\title{
Transferts rivière-nappe et effet filtre des berges Application aux transferts de l'azote
}

\author{
Claude Doussan $\left({ }^{1}\right)^{*}$, Emmanuel Ledoux $\left({ }^{1}\right)$, Guillemette Poitevin $\left({ }^{1}\right)$, Michel Detay $\left({ }^{2}\right)$ \\ (1) Centre d'Informatique Géologique, Ecole de Mines de paris, Fontainebleau, France \\ $\left(^{2}\right)$ Lyonnaise des Eaux, Cholet, France
}

\section{Introduction}

Dans de nombreux pays, et plus particulièrement en Europe, les aquiferes alluviaux sont exploités de façon intensive pour la production d'eau potable. En effet, ces aquiferes présentent généralement un ensemble de caractéristiques très favorables à l'exploitation: faible profondeur, productivité élevée, adéquation (au niveau géographique) entre la production et l'utilisation, bonne qualité de l'eau pompée...

Ainsi, ce mode d'exploitation représente plus de la moitié du volume des eaux souterraines extraites en France [1], environ $40 \%$ en Hongrie [2], et plus de $80 \%$ en Suisse [3].

Un trait essentiel de ce type de nappe est la liaison privilégiée qui existe entre celle-ci et le cours d'eau qui la traverse. En situation naturelle (c'est-à-dire sans captage), le cours d'eau draine, le plus souvent, la nappe. Cette situation s'inverse temporairement lors des crues. Quand des captages sont implantés dans l'aquiferre à proximité de la berge, les échanges ne s'effectuent alors que dans le seul sens rivière-nappe du fait de la mise en dépression de la nappe par les pompages. Cette situation hydraulique est appelée

(*) Actuellement : INRA, Unité de Science du Sol, Domaine Saint Paul, Site Agroparc, 84914 Avignon cedex, France. Claude Doussan a obtenu le premier prix Henri Milon.
« réalimentation induite». Cette caractéristique est à l'origine du renouvellement de la ressource en eau de l'aquifere (au détriment de la rivière) et de la forte productivité afférente.

L'eau prélevée au forage est donc un mélange d'eau de nappe, au sens strict, et d'eau issue de la rivière. Cette dernière composante peut représenter de 70 à $100 \%$ de l'eau pompée pour des forages situés à faible distance du cours d'eau[4]. On constate également que l'eau ainsi produite, et c'est une caractéristique essentielle de ce type d'exploitation, présente des modifications physico-chimiques considérables par rapport à l'eau superficielle dont elle est issue. Cet effet est connu sous les appellations « effet filtre des berges * ou \& filtration par les berges ". Il est le plus souvent perçu comme un effet positif car généralement nombre de polluants présents dans la rivière ne sont pas retrouvés dans l'eau extraite $[5,6]$.

Différents mécanismes interviennent dans cet effet filtre des berges. Ce peuvent être des processus de type mécanique (filtrations physiques des particules, des bactéries et virus... [7, 4]), de type hydrodynamique (transfert convecto-dispersif, dilution $[8,9]$ ). des phénomènes biologiques relatifs à l'activité de la microflore des sédiments de la rivière ou de l'aquifere (minéralisation de la matière organique dissoute provenant du fleuve, dégradation de composés organiques d'origine anthropique tels que des hydrocarbures, des pesticides... $[10,11,12])$ ou encore des processus physico-chimiques (adsorption. précipitation, réactions redox... $[13,14,15])$.

Assez paradoxalement, malgré l'intérêt qualitatif et quantitatif de ce type d'exploitation, relativement peu d'efforts ont été faits pour rationaliser l'implantation des captages vis-à-vis de cet effet filtre. Ce point est d'autant plus important que les ressources d'eau superficielles sont actuellement soumises à de nombreux types de pollutions qui peuvent être chroniques (pollution diffuse par les nitrates par exemple) ou plus ponctuelles (hydrocarbures, micropolluants organiques...). Ainsi, dans le cas des nitrates, les captages implantés dans les plaines alluviales soumises à une agriculture intensive, présentent très souvent une contamination par des nitrates d'origine agricole. En revanche, les forages situés en bordure de rivière montrent généralement une concentration en nitrate faible, inférieure à celle trouvée dans le cours d'eau, visualisation directe d'une des conséquences de l'effet filtre des berges. Le mélange des eaux des différents forages permet alors de produire une eau brute, destinée à la consommation, respectant les normes de potabilité. Cependant, en cas d'augmentation de la concentration en nitrate dans le cours d'eau (par nitrification des effluents de station d'épuration par exemple), il est nécessaire de savoir si l'effet filtre des berges permet une protection efficace, et stable dans le temps, de ces captages qui puisse éviter une contamination de l'ensemble du champ captant.

River - Groundwater relationships and bank filtration : an emphasis on nitrogen species behavior and evolution

In this article, the authors present results of a study designed to investigate hydrodynamical and geochemical environment of bank filtration. The experimental site is a well field located along the Seine river, downstream of Paris (France). At this place, hydrodynamical and geochemical features have been considerably altered from natural background by man action (intensive pumping in the aquifer, chronical pollution of the Seine river by sewage of treatment plant or by agriculture). Nitrogen species behavior during bank filtration was more particularly examined in the experimental site.

Water issued from bank filtration shows drastic changes when compared to river water, denoting a reductive trend (drastic decrease of oxygen, nitrate, sulfate concentrations and increase in ammonium, iron... concentrations). This geochemical evolution of the water in the aquifer is the reflect of intensive bacterial mineralization which happens in the first few meters of filtration through river bed sediments. As a consequence for nitrogen species, denitrification is very effective and no nitrate is found below a few centimeters depth in the sediments. On the contrary, ammonium concentration drastically increases at the same time. Away from the river bed sediments, the aquifer mainly acts as a buffer toward physico-chemical variations owing to processes like dilution, adsorption or precipitation/dissolution.

The problem of ammonium transfer to the well is adressed and key parameters, as percolation velocity and organic carbon content of sediments, are discussed in conjunction with drinking water production using bank filtration. 
Dans cette optique, nous présentons dans cet article quelques données expérimentales relatives à la filtration par les berges dans le cas d'un champ captant situé le long de le Seine, en aval de Paris. Ces données nous permettront de mieux cerner l'origine de cet effet filtre, ainsi que le contexte biogéochimique qu il entretient. tout en en détaillant plus particulièrement le comportement de l'azote dans ce système. Sur ces bases, un modèle décrivant les grands traits de l'évolution de la composition des caux au cours de la filtration par les berges a été construit et nous permettra de dégager les paramètres clef affectant les transformations des formes azotées lors du transfert rivière-nappe.

\section{Méthodes et moyens}

\subsection{Le site expérimental}

Pour une description détaillée du site et des méthodes. on pourra se reporter à [16] et 1171. Le site expérimental retenu est une ile située sur la Seine qui fait partie du champ captant de Flins-Aubergenville exploité par la Lyonnaise des Eaux (fig. I). Le champ captant. situé à une trentaine de kilomètres à louest de Paris et produisant $45.10^{6} \mathrm{~m}^{3} \mathrm{~d}$ eau annuellement. est un des plus importants de la région parisienne [18]. Ce site se trouve dans un contexte particulier car l'environnement hydrique est ici profondément modifié par l'activité humaine, que ce soit au niveau des écoulements (régulation de la Seine. modification de l'hydrodynamique de la nappe du fait des pompages intensifs) ou au niveau géochimique (pollution chronique de la Seine. en aval de la station d'épuration d'Achères dans ce secteur. influence de l'agriculture...). L'eau extraite au forage installé sur l'île (forage A8, qui débite environ $150 \mathrm{~m}^{3} / \mathrm{h}$ ) provient quasiment exclusivement de la Seine. L'aquifère est ici constitué de craic fissurée recouverte d'alluvions sablosilteuses (fig. 2). A la suite d'une étude hydrogéologique préliminaire, un ensemble de piézomètres a été installé le long d'une ligne de courant entre la Seine et le forage. II est ainsi possible d'échantillonner de l'eau dans les différents horizons de l'aquifêre et donc de suivre les composés en cours de filtration (fig. 2). Le dispositif permet également la mesure des températures et des charges hydrauliques dans la nappe.

\subsection{Méthodes d'échantillonnage et d'analyse}

Des prélèvements d'eau de Seine ou de la nappe ont été effectués pendant un an avec une cadence mensuelle ou bimensuelle. Les prélèvements d'eau de nappe sont effectués dans les piézomètres grâce à une pompe immergée. Les paramètres oxygène, conductivité. $\mathrm{pH}$. potentiel rédox sont mesurés sur le terrain avec une cellule de circulation branchée en sortic de pompe alors que les éléments majeurs. les diverses formes de l'azote, ainsi que les métaux fer et manganèse ont été dosés par le laboratoire d'analyse de la Lyonnaise des Eaux (CIRSEE) La caractérisation physico-chimique des sédi-

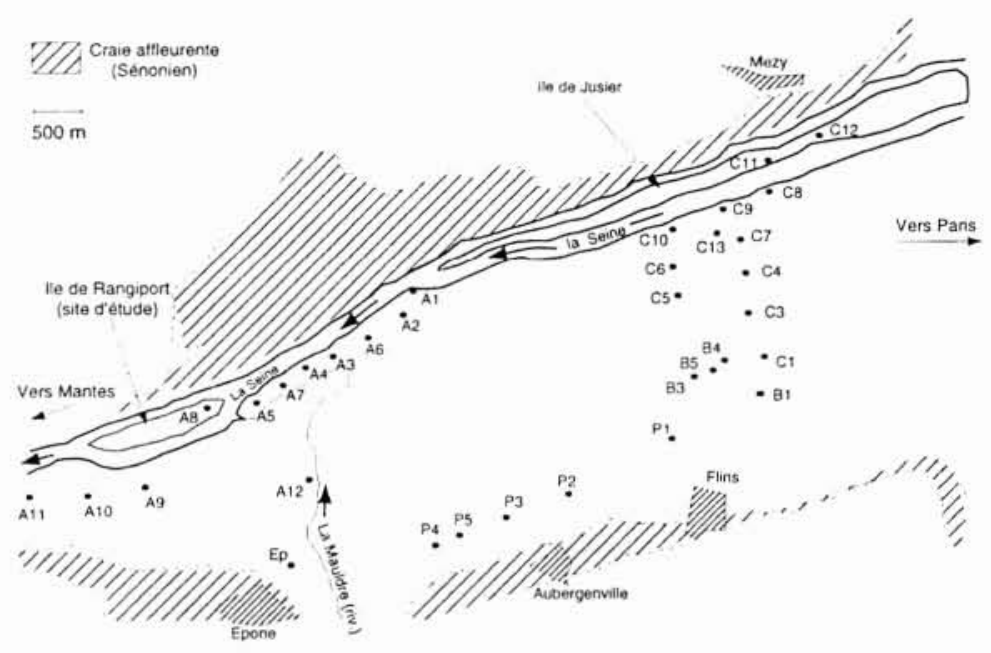

A 1. Situation géographique du champ captant et localisation des forages AEP.

ments superficiels de la Seine a été entreprise a l'aide de carottages et de sondes à diffusion [19]. Les diverses formes de l'azote, le carbone organique (dissous et particulaire) et parfois fer. manganèse et phosphate ont été analysés sur l'eau interstitielle. Un carottier a été spécialement construit pour pouvoir recueillir des sédiments en zone profonde de la Seine.

Lors du forage des piézomètres, des échantillons de craie et d'alluvions ont été prélevés

de façon aseptique pour être utilisés dans des expériences en batch de dénitrification ou de comptage bactériens. Ils ont été conservés sous azote et en chambre froide, les manipulations débutant quelques jours plus tard. Les analyses menées en laboratoire sur les sédiments de l'aquifère et de la Seine comprenaient un suivi de la dénitrification. en batch. par blocage de la réaction au stade $\mathrm{N}_{2} \mathrm{O}$ par ajout d'acétylène. Le protoxyde d'azote produit est mesuré par chromatographie en
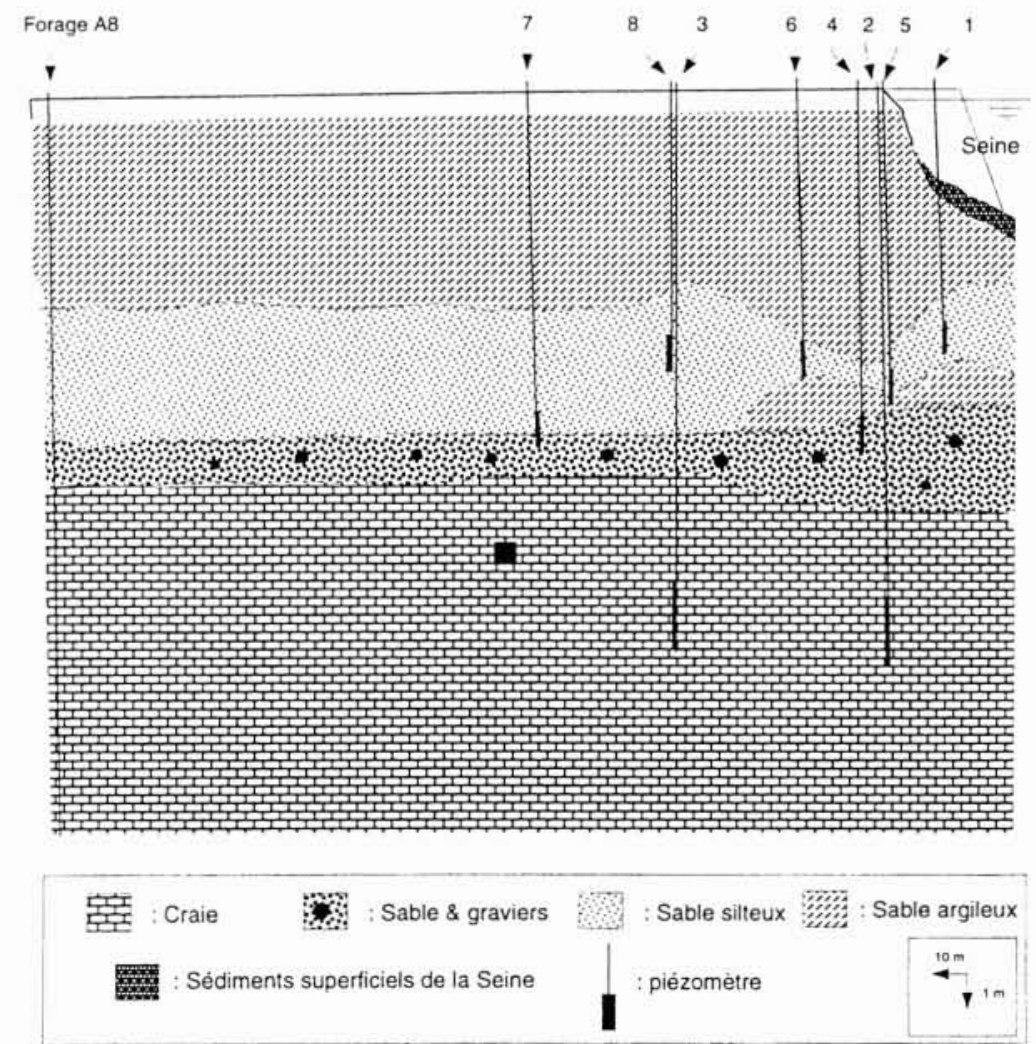

A 2. Coupe géologique du site expérimental et installation du dispositif piézométrique. 
phase gazeuse. Diverses expérimentations sur l'évolution des formes ioniques dissoutes de l'azote ont également été effectuées avec des essais en batch.

Pour caractériser l'hydraulique du système, les charges piézométriques et la température dans les piézomètres et en Seine ont été relevées pendant l'année de mesure. Un essai de traçage à la fluorescéine a également été réalisé.

\section{Résultats}

\subsection{Hydrodynamique des transferts rivière-nappe}

La définition des zones d'infiltration de la Seine et du trajet de l'eau dans l'aquifère sont nécessaires pour appréhender correctement l'évolution de la composition de l'eau lors du transfert rivière-nappe. Il est mis en évidence que le transfert de l'eau dans l'aquifere se déroule essentiellement dans les dix premiers mètres de craie fracturée, la contribution des alluvions sus-jacentes étant négligeable. Un important colmatage des berges (lié à l'alimentation induite) est souligné par l'existence d'une perte de charge d'environ $3 \mathrm{~m}$ entre la Seine et les premiers piézomètres situés à $5 \mathrm{~m}$ de la rive. Ces sédiments colmatants peuvent être plus ou moins décapés lors des épisodes de crue de la Seine, mais un retour aux conditions d'origine s'effectue dès que le débit faiblit.

Un point important mis en évidence, grâce à l'utilisation conjointe des données thermiques et hydrauliques acquises sur le site dans une modélisation hydrodynamique du système, est l'hétérogénéité des perméabilités des sédiments du lit du fleuve [20]. En effet, les sédiments du centre de la Seine sont environ quinze fois plus perméables que ceux situés en proximité de berge. On obtient ainsi une répartition des vitesses d'infiltration qui augmente vers le centre de Seine : de 5 à $10 \mathrm{~mm} / \mathrm{j}$ sur le bord pour quelques $150 \mathrm{~mm} / \mathrm{j}$ plus au centre (fig. 3). Ce contraste de vitesse est un point essentiel pour l'évolution des composés en cours de filtration, comme nous le verrons par la suite.

Au travers de la trame dynamique que constitue I'hydraulique du système rivièrenappe, nous allons voir à présent les caractéristiques générales de l'évolution géochimique de l'eau au cours du transfert.

\subsection{Caractérisation physico-chimique des transferts rivière-nappe}

A un niveau global, la composition chimique des eaux de la nappe montre un changement radical par rapport aux eaux de la Seine dont elles sont issues. Ce changement conduit à l'installation de conditions sévèrement réductrices, permanentes dans le temps, de la berge jusqu'au forage. Ces conditions sont toutefois plus marquées à proximité de la berge.

Ainsi, et par rapport à la Seine, nitrate, nitrite et oxygène ont complètement disparu dans l'aquifere tandis que la concentration en

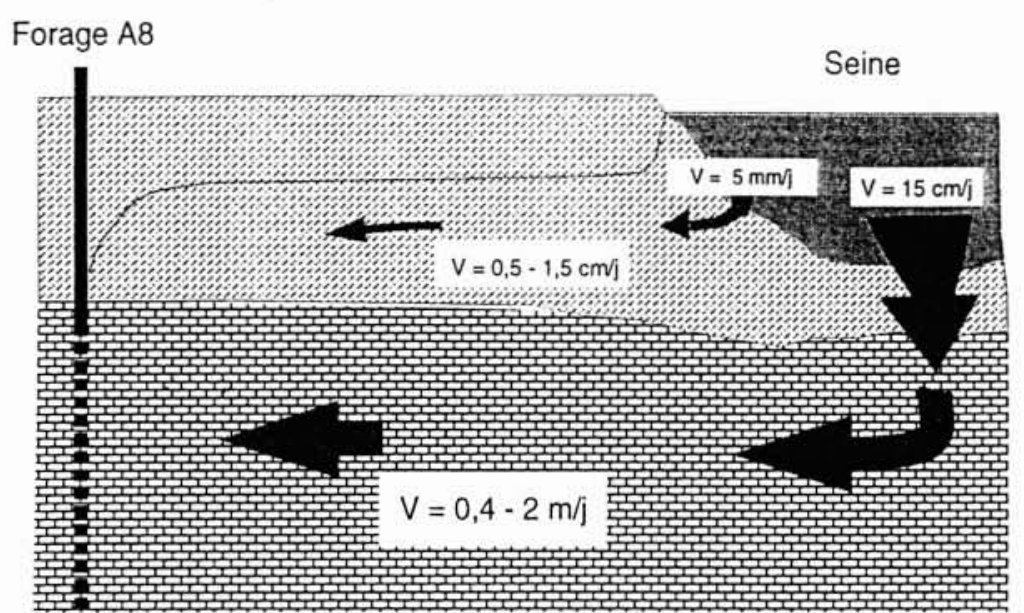

A 3. Vitesses d'écoulement entre la rivière et le forage sur le site expérimental.

sulfate chute de moitié (fig. 4). Au contraire, ammonium, fer, manganèse, hydrogène sulfuré et hydrogénocarbonate se présentent dans l'aquifère à des concentrations très supérieures à celles de la Seine, et ce dès les premiers piézomètres en berge. Ceci montre qu'il existe un front de concentration entre la Seine et les premiers piézomètres. Cette zone réductrice apparaît comme un phénomène général et a été décrite sur d'autres sites de
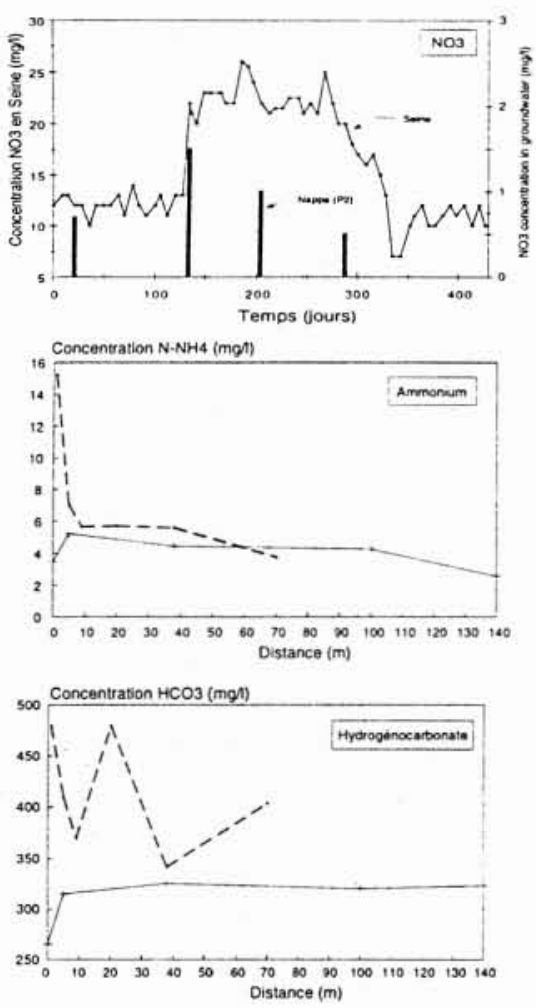

réalimentation induite $[4,21,22]$. L'intensité réductrice est cependant ici relativement forte, comparée à ces autres sites, comme en témoignent les concentrations en fer (de 2 à $8 \mathrm{mg} / \mathrm{l}$ ) ou en ammonium (de 5 à $20 \mathrm{mg}$ $\left.\mathrm{NH}_{4} / 1\right)$

A l'intérieur de l'aquifere, les profils de concentration de la plupart des composés analysés varient peu, soulignant un rôle « tampon " de l'aquifere vis-à-vis des modifi-
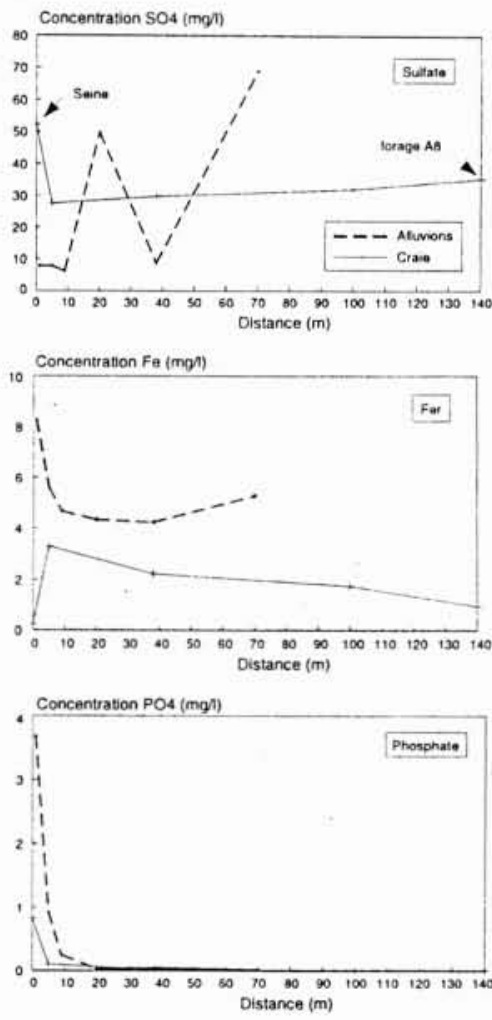

4. Evolution géochimique entre la Seine et la nappe : a) Evolution temporelle des nitrates en Seine et dans un piézomètre situé à $5 \mathrm{~m}$ de la rive (temps $=0$ le 03/07/90) - b, c, d, e, f) Profils de concentration en sulfate, ammonium, fer, hydrogénocarbonate et phosphate entre la Seine et le forage A8 (valeurs moyennes sur l'année de mesure). 
cations physico-chimiques. Ce comportement est à relier à des phénomènes de précipita tion/dissolution (cas du fer et des phosphates). d'adsorption (ammonium) ou de dilution (chlorure, sodium).

L'évolution chimique globale de l'eau en cours de filtration entre la Seine et les pre miers piézomètres en berge peut être représentée par un modèle biogéochimique simple (balance de masse basée sur les variations d'alcalinité et de carbone total) qui permet de définir et quantifier les réactions majeures se déroulant lors du transfert à l'état permanent. Ce sont :

- la dissolution/ précipitation de calcite et l'altération des silicates.

- la minéralisation de la matière organique avec :

- la respiration aérobie et dénitrification.

- la sulfato-réduction.

- une activité méthanogène.

Ce calcul met également en évidence. et ceci est un point important. que cette activité minéralisatrice bactérienne ne peut être soutenue que par la présence de carbone organique particulaire dans le sédiment. l'apport en carbone organique dissous en provenance du fleuve étant insuffisant.

Du point de vue de l'évolution et du transfert des composés azotés, une dénitrification totale est observée dès les premiers piézomètres implantés en berge (fig. 4). des nitrates n'apparaissant dans l'aquifere que de façon fortuite à la faveur d'infiltrations provenant des sols superficiels (champ cultivé). De plus. les expériences in vitro ont montré la capacité potentielle des alluvions et de la craie à la dénitrification. Ces capacités mesurées sont, pour la craic, de l'ordre de 0.1 à $0.3 \mathrm{nmol} \mathrm{N} / \mathrm{g} / \mathrm{h}$ et pour les alluvions susjacentes de 0.5 à $1,5 \mathrm{nmol} \mathrm{N} / \mathrm{g} / \mathrm{h}$. Cette activité dénitrifiante des alluvions, anaérobies en conditions naturelles, limite le flux de nitrates en provenance des sols cultivés superficiels.

Une analyse de l'évolution de l'azote minéral dans l'aquifere, remontant au début de la mise en exploitation du forage. montre que la capacité dénitrifiante des berges s'était mise en place dès l'installation d'une réalimentation induite. Il ressort également que le maintien de conditions réductrices et la formation d'ammonium au sein de l'aquifere sont directement liés au niveau de la surface piézométrique de la nappe qui conditionne les possibilités de réoxygénation de celle-ci.

\subsection{Les sédiments du fleuve: une interface biogéochimique active entre la rivière et la nappe}

L'ensemble de ces analyses montre que ce sont tout d'abord les sédiments superficiels du lit de Seine, interface entre le fleuve et la nappe, qui vont conditionner la dynamique des composés en cours de filtration. Ces sédiments montrent en effet tous les signes d'une activité minéralisatrice intense de la matière organique. Couplée à ce processus de minéralisation. la dénitrification y est particulièrement efficace et on observe une disparition des nitrates dans les premiers centimètres de sédiments. Cette activité dénitrifiante est corroborée par des taux de dénitrification mesurés en laboratoire qui se trouvent dans la fourchette de $1.5 .10^{-7}$ a $4 \cdot 10^{-7} \mathrm{~mol} \mathrm{~N} / \mathrm{g} / \mathrm{h}$ avec des concentrations $\mathrm{NO} / \mathrm{l}$. Cette activité dénitrifiante chute avec la distance dans le sédiment, pour s'annuler vers $30 \mathrm{~cm}$ de profondeur (fig. 5 ).

C'est l'hydrolyse exoenzymatique de la matière particulaire présente dans le sédiment qui fournit des quantités importantes en carbone organique dissous (de 50 à $100 \mathrm{mg} / \mathrm{l}$ ) disponibles pour l'activité bactérienne. $\mathrm{Ce}$ carbone étant excédentaire par rapport aux accepteurs d'électrons minéraux, nitrate et sulfate sont consommés par les mécanismes de respiration anaérobie (fig. 6). La minéralisation peut alors être poussée jusqu'au stade extrême de la méthanogenèse. Dans ces conditions, on arrive à la formation d'un milieu fortement réduit avec mise en solution de quantités importantes d'ammonium, fer et phosphate (fig. 6).

Au niveau des sédiments du centre de la Seine, moins riches en matière organique et où la vitesse de percolation de l'eau est plus rapide. les mêmes processus apparaissent en étant toutefois plus atténués (fig. 6).

$\mathrm{Ce}$ sont donc les mécanismes de minéralisation de la matière organique, qui se déroulent dans le premier mètre d'infiltration, qui conditionnent la qualité et la composition globale de l'eau retrouvée par la suite dans l'aquifere (absence de nitrate. présence de fer. ammonium...) et ce d'autant plus, du fait d'un niveau piézométrique bas qui limite les possibilités de réoxygénation de l'aquifere à partir de la surface.

La production importante d'ammonium dans l'eau interstitielle de ces sédiments du fond de la Seine (de 30 à $300 \mathrm{mg} / \mathrm{l}$ ) pose le problème de son devenir et de son transfert vers les captages. On observe, actuellement. que les quantités retrouvées dans la nappe sur l'île (de $4 \mathrm{mg} / \mathrm{l}$ au forage à $20 \mathrm{mg} / \mathrm{l}$ à proximité des berges) sont nettement inférieures à celles retrouvées dans les sédiments. L’ammonium est donc retardé dans le milieu poreux pendant son transfert des sédiments superficiels vers la berge. Des expériences ambiantes en nitrates de l"ordre de $10 \mathrm{mg}$ -

d'adsorptions conduites en laboratoire montrent, en effet, une rétention de l'ammonium par la matrice solide. Les coefficients de partage mesurés sont de l'ordre de 1 à $4 \mathrm{ml} / \mathrm{g}$ dans les alluvions et sédiments et de $48 \mathrm{ml} / \mathrm{g}$ dans la craie. De plus, dans le cas de la craic en place, il existe une possibilité de diffusion dans la matrice qui retarde aussi le transport.

Une régulation des concentrations en ammonium peut également s'opérer par précipitation de minéraux ammoniacaux. en particulier de tanarakite (phosphate mixte d'aluminium et ammonium). Pour cela, des conditions de $\mathrm{pH}$ relativement faible $(=6,5)$ et de concentration en phosphate forte $(\simeq 1 \mathrm{mg} / \mathrm{l})$ doivent être remplies. Cependant. même si les sédiments sont susceptibles de réunir les conditions thermodynamiques nécessaires à la précipitation, seul un examen minéralogique pourrait affermir cette hypothèse (problèmes de cinétique, d'inhibition). Une simulation simplifiée du transfert de l'ammonium, utilisant des coefficients d'adsorption mesurés en laboratoire, montre que, dans le cas particulier du site que nous avons étudié, le front de concentration atteindrait actuellement la partie supérieure de la craie située sous la Seine.

\subsection{Quelques paramètres essentiels pour l'évolution des formes de I'azote dans le système rivière- nappe}

Dans le cadre de la problématique sur les transferts d'azote dans le système rivièrenappe, et sur la base des données expérimentales obtenues dans cette étude, nous avons focalisé la conceptualisation de l'effet filtre des berges sur la percolation de l'eau du fleuve dans le premier mètre de sédiments. Un modèle conceptuel débouchant sur un modèle numérique ont été bâtis pour représenter. entre autres, l'évolution des formes de l'azote dans les sédiments en couplant des processus physiques: transferts diffusifs et convectifs. et bactériens de minéralisation de la matière organique : respiration aérobie, dénitrification, sulfato-réduction, méthanogenèse [23].

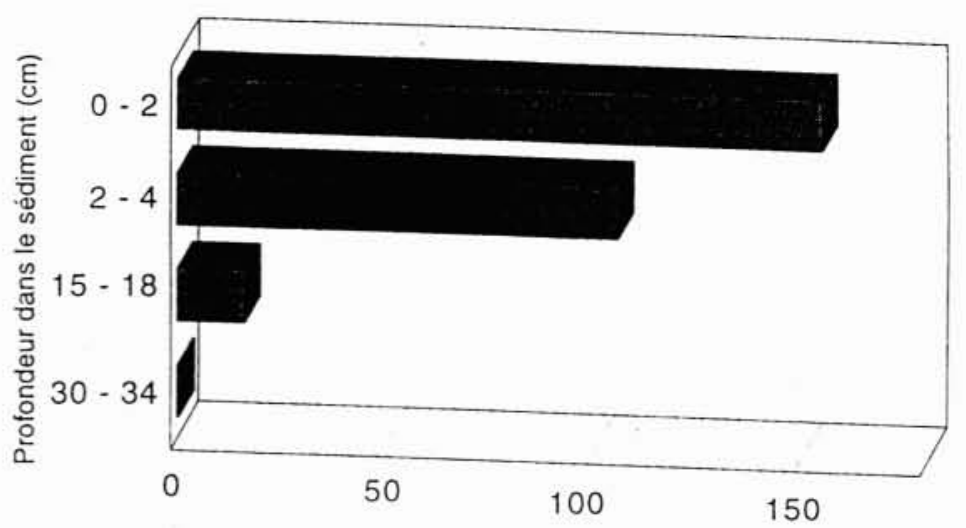

Vitesse initiale de dénitrification ( $\mathrm{nmol} \mathrm{N} / \mathrm{g} / \mathrm{h}$ )

5. Vitesses de dénitrification dans les sédiments de la Seine mesurées en batch par blocage à l'acétylène. 

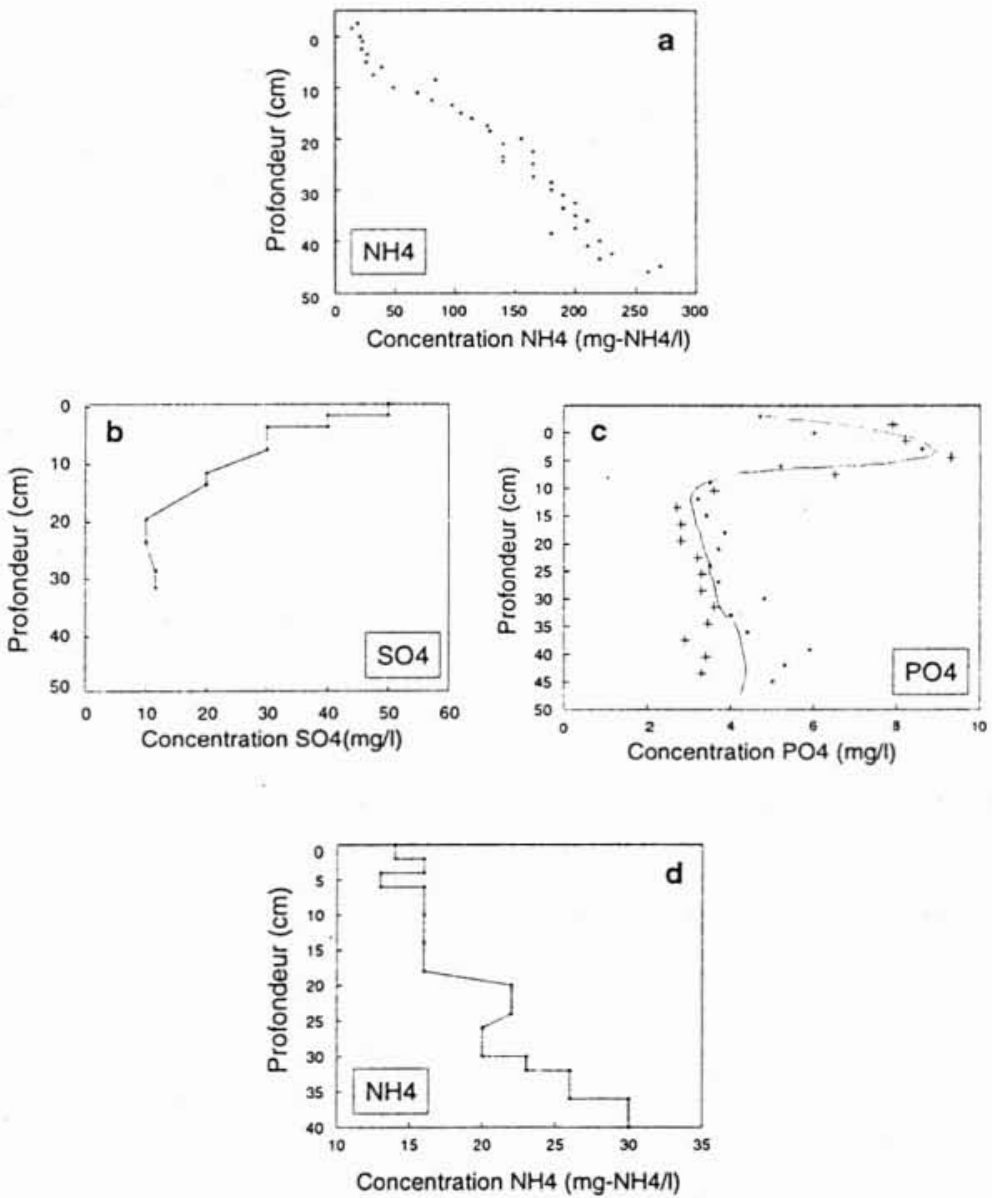

A 6. Profils de concentration dans les sédiments de la Seine : $-a, b, c)$ ammonium, sulfate, et phosphate dans les sédiments situés à proximité de la rive - d) ammonium dans les sédiments situés au centre de la Seine.

Ce modèle couplé permet de reproduire de façon satisfaisante les expériences en colonne de von Gunten et Zobrist [24] dans lesquelles différents accepteurs d'électrons sont utilisés dans l'oxydation d'un substrat organique.

Nous avons alors appliqué cette modélisation aux caractéristiques des sédiments du site d'étude, sur la base de paramètres déduits d'expériences en laboratoire ou tirés de la littérature. Les principaux phénomènes de la filtration par les berges sur ce site, tels qu'une dénitrification efficace, la consommation des sulfates, ou la forte production d'ammonium sont bien restitués par la modélisation. On retrouve, en particulier, un front de nitrate qui ne pénètre que de quelques centimètres dans le sédiment.

Une étude de sensibilité a montré que deux paramètres sont essentiels dans l'évolution du filtrat. Ce sont :

- la vitesse de percolation de l'eau dans les sédiments,

- la quantité de carbone organique particulaire (et les possibilités de solubilisation de celui-ci).

La vitesse de percolation, quand elle croît, permet une pénétration plus importante des accepteurs d'électrons dans le sédiment
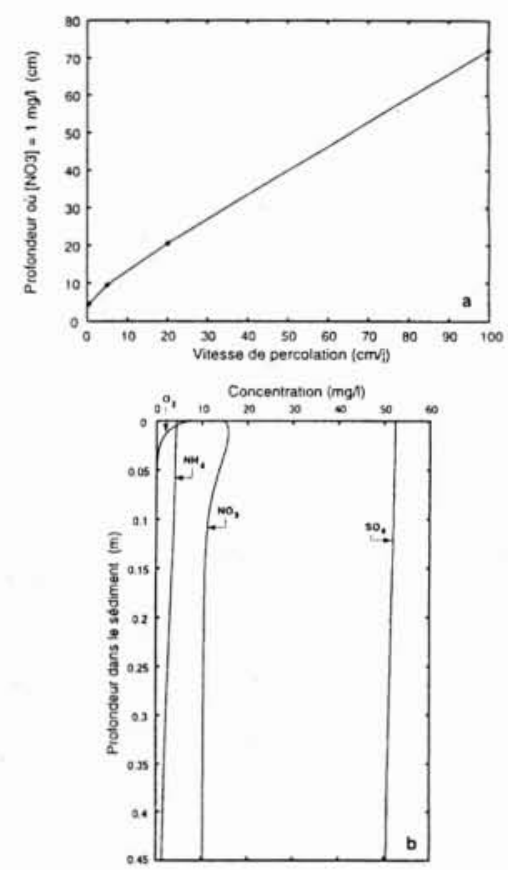

A 7. Modélisation des transferts : a) Exemple simulé de l'effet de la vitesse de percolation de l'eau dans les sédiments sur la profondeur de pénétration des nitrates. - b) Exemple simulé d'un cas où il n'y a pas de carbone organique particulaire dans le sédiment (à comparer avec les profils en ammonium et sulfate de la figure 6-a et b).

l'eau issue, de façon indirecte, de la rivière. Au niveau géochimique, le transfert se traduit par une modification radicale de la composition de l'eau pendant son trajet conduisant à l'installation de conditions réductrices dans l'aquifère (chute des teneurs en oxygène. nitrate, ... augmentation de celles en ammonium, fer...).

Cette évolution, et donc la qualité de l'eau pompée, va dépendre, en grande partie, des interactions entre la vitesse de percolation et la minéralisation du carbone organique dans les premiers mètres de filtration. Dans ces conditions, il semble que des vitesses d'infiltration de quelques mètres par jour, qui dépendent de la distance du forage à la berge, dans des sédiments contenant de l'ordre de $2 \%$ en matières organiques, permettent d'arriver à une dénitrification poussée de l'eau pompée sans établissement de conditions réductrices trop sévères qui impliqueraient la formation de fer et d'ammonium. Ces valeurs sont en bon accord avec les quelques données relatives à d'autres sites expérimentaux de filtration par les berges placés dans des contextes différents. Ainsi, pour un site bien étudié situé sur la rivière Glatt en Suisse $[12,9,21]$ les sédiments présentent une teneur en matière organique inférieure à $0,5 \%$ tandis que le flux de percolation est de l'ordre de $2 \mathrm{~m} / \mathrm{j}$. On trouve ici que 
la concentration en nitrate est de l'ordre de $5 \mathrm{mg} / \mathrm{l}$ dans l'aquifère pour $30 \mathrm{mg} / \mathrm{l}$ dans la rivière. L'ammonium, pour sa part, présente une concentration inférieure à $0.3 \mathrm{mg} / \mathrm{l}$ dans la nappe. En France, sur un site jouxtant le Rhône décrit par Ille [22]. la teneur en carbone organique des sédiments atteint I \% tandis que les vitesses de percolation sont comprises entre 4 et $6 \mathrm{~m} / \mathrm{j}$. Ici, de même, la concentration en nitrate est faible dans la nappe $(0$ à $1 \mathrm{mg} / \mathrm{l}$ près des berges pour 5 à $10 \mathrm{mg} / \mathrm{l}$ dans le fleuve), de même que la concentration en ammonium (de l'ordre de $0,3 \mathrm{mg} / \mathrm{l}$ ). Dans le cas du site présenté dans l'article, les vitesses de percolation, varient entre $5 \mathrm{~mm} / \mathrm{j}$ et $15 \mathrm{~cm} / \mathrm{j}$ pour des teneurs en carbone organique dans le sédiment supérieures à $1 \%$. Si on ne retrouve pas de nitrate dans l'aquifere (la concentration en Seine oscillant entre 10 et $25 \mathrm{mg} / \mathrm{l}$ ), la nappe présente des teneurs élevées en ammonium (de 6 à $20 \mathrm{mg} / \mathrm{l}$ ) près des berges.

Pour terminer et reprendre le problème du nitrate posé dans l'introduction, on peut noter que, paradoxalement, l'apport en carbone organique dans le fleuve peut avoir un effet " bénéfique " du point de vue de la filtration par les berges. Ainsi, une augmentation importante de la teneur en nitrates dans le fleuve (à $50 \mathrm{mg} / \mathrm{l}$ par exemple, qui est la norme de potabilité) ne devrait pas affecter le captage. En effet, la saturation des capacités dénitrifiantes du sédiment est loin d'être atteinte si un renouvellement du carbone organique est maintenu. Dans le cas du site étudié, cet apport est assuré en Seine par la sédimentation en carbone particulaire qui provient pour une large part des stations d'épuration situées en amont. Ici, cependant, le flux de carbone excédentaire conduit à l'établissement de conditions réductrices peu favorables pour la qualité des eaux issues de la filtration par les berges en ce qui concerne l'ammonium et le fer par exemple.

Remerciements : Cette étude a bénéficié d'un soutien financier de l'Agence de l'Eau SeineNormandie.

\section{Références}

[1] Castany G. (1985), - Liaisons hydrauliques entre les aquiferes et les cours d'eau. Stygologia. 1(1), pp. 1-25.

[2] Laszlo F., Hommonay Z.S. (1986). Study of effects determining the quality of bank filtered water. Conjunctive Water Use, IAHS pub. n 156, pp. 181188.
[3] vON GUNTEN H.R., KULL T.P. (1986), Infiltration of inorganic compounds from the Glatt river, Switzerland, into a groundwater aquifer. Water. Air and Soil Pollution, 29, pp. 333-346.

[4] Bize J., Grenet B.. Maneglier H. (1981). - Le pouvoir épurateur du complexe alluvial en bordure de rivière. Techniques et Sciences Municipales L'eau, juillet, pp. 393-401.

[5] Bourg A. (1992), - Effet filtre des berges et qualité des eaux pompées en nappe alluviale. Courants, 14, pp. 14-19.

[6] Sontheimer H. (1980), - Experience with river bank filtration along the Rhine river. Journal $A W W A$, juillet, pp. 386-390

[7] VITUKI (1983). - Water quality problems of the bank filtered water supply. The Hungarian experience : United Statement by the Research Centre for Water Resources Development (VITLKI), Budapest, Hungary for the working group on bank well filtration, Budapest, 20-24 February 1983, $20 \mathrm{p}$.

[8] DufFy J.C.. Gelhar L.W. (1986). - A frequency domain analysis of groundwater quality fluctuations : interpretation of field data. Water Resources Research, 22(7), pp. 1115-1128.

[9] HoEhn E.. Zobrist J., SchwarzenbaCh R.P. (1983), - Infiltration von flusswasser ins grundwasser. Hydrogeologische und hydrochemische untersuchungen im Glattal. Gas-WasserAbwasser, 63 (8), pp. 401-410.

[10] Schaffner C. Ahel. M., Giger W (1985). - Behavior of organic micropollutants during infiltration of river water into groundwater : Results of field study in Glatt valley, Switzerland; in Organic Micropollutants in the aquatic Environment, Proceedings of the fourth european symposium, Vienna, Austria, 22-24 Oct., Reidel Publishing company. pp. $150-153$

[11] KUHN E., VAN LOODSDRECHT M., Giger W., SchWarzenbach R.P. (1987), Microbial degradation of nitriloacetate (NTA) during river groundwater infiltration : Laboratory Column studies. Water Research, 10, pp. 1237-1248.

[12] SChWARZEnbach R.P., Giger W.. HOEHN E., SCHNEIDER J.K. (1983). Behavior of organic compounds during infiltration of river water to groundwater - field studies. Environmental Science and Technology, 17, pp. 472-479.

[13] DARMENDRAIL D. (1987). - Filtration géochimique des métaux lors de leur transfert d'une rivière vers une nappe alluviale. Application à la nappe des alluvions du Rhône et de la Deule. Thèse de doctorat, Université de Bordeaux III, 453 p.
[14] VON GUNTEN H.R., LIENERT C.H. (1993). - Decreased metal concentrations in groundwater caused by controls of phosphate emissions. Nature, 364(6434). pp. $220-222$

[15] Brauch H.J., KuHN W. (1986). Behavior of organic micropollutants during river bank filtration and drinking water treatment ; in behavior and trans formation of organic pollutants in groundwater treatments. CEE Water Pollution Research Report, rep EUR 11094, pp. 13-32.

[16] Doussan C. (1994). - Transferts rivière-nappe et effet filtre des berges : application aux transferts de l'azote. Thèse de doctorat de I'E.N.S.M.P. Mémoire des Sciences de la terre, $\mathrm{n}^{\circ} 23$. $210 \mathrm{p}$.

[17] Portevin G. (1994), - Etude des mécanismes de transfert des composés minéraux de l'azote d'un cours d'eau vers sa nappe alluviale. Thèse de doctorat, Université Paris VI, 182 p.

[18] Detay M., D’Arras D., Suzanne P. (1992), - La gestion des eaux souterraines en région parisienne ouest. $\mathrm{La}$ Houille Blanche, 4, pp. 295-298.

[19] HessLein R.H. (1976). - An in siu sampler for close interval pore water studies. Limnology \& Oceanography, 21, pp. $912-914$

[20] Doussan C.. Toma A.. Paris B.. Portevin G., Ledoux E.. Detay M. (1994). - Coupled use of thermal and hydraulic head data to characterize river - groundwater exchanges. Journal of Hydrology: 153, pp. 215-229.

[21] JaCOBS L.A., von GunTEN H.R., KeIL R., KusLys M. (1988), - Geochemical changes along a river groundwater infiltration flow path: Glattefelden, Switzerland. Geochemica \& Cosmochimica Acta, 52, pp. 2693-2706.

[22] lLLE C. (1992). - Interface rivièrenappe : caractérisation physique et chimique, rôle protecteur de l'aquifere. Cas de l'île du Grand Gravier. Thèse de doctorat, Université Grenoble I, $155 \mathrm{p}$.

[23] Doussan C., Poitevin G., Ledoux E. DETAY M. (1995). - River bank filtration : modeling of the changes in water chemistry with emphasis on nitrogen species. Soumis à Journal of Contaminant Hydrology.

[24] von GunTEN U., ZoBRIST J. (1993). Biogeochemical changes in groundwater infiltration systems : column studies. Geochemica \& Cosmochemica Acta, 57 pp. 3895-3906. 